\title{
A note on global dynamics and imbalance effects in the Lucas-Uzawa model*
}

\author{
R. Boucekkine $^{\dagger} \quad$ B. Martínez J. R. Ruiz-Tamarit $^{\ddagger}$
}

September 2007

\begin{abstract}
In the traditional literature on the Lucas-Uzawa model, it is proved that in the neighborhood of the long-run balanced growth path, human capital stock grows at a rate greater than its long-run counterpart when the ratio physical to human capital is above its long run value if and only if the capital share in the production of physical good is lower than the inverse of the elasticity of intertemporal substitution in consumption. We first prove that the claim is true outside the neighborhood of balanced growth paths. More importantly, we identify a crucial asymmetry: whatever the position of the capital share with respect to the inverse of the elasticity of intertemporal substitution, physical capital stock always grows at a rate lower than its long-run counterpart when the ratio physical to human capital is above its long run value.
\end{abstract}

Keywords: Lucas-Uzawa, hypergeometric functions, imbalance effects, global dynamics.

Journal of Economic Literature: E20, O40, C60.

${ }^{*}$ R. Boucekkine acknowledges the support of the Belgian research programme ARC 03/08-302. J. R. Ruiz-Tamarit acknowledges the financial support from the Spanish CICYT, Project SEJ-200404579/ECON, and the Grant PR2006-0294 from the Secretaría de Estado de Educación y Universidades, Spanish MEC. B. Martínez acknowledges the financial support of the Spanish Ministerio de Educación y Ciencia, Research Project SEJ2005-02829/ECON.

${ }^{\dagger}$ Corresponding author. CORE and Department of Economics, Université Catholique de Louvain (Belgium), and Department of Economics, University of Glasgow (UK). Place Montesquieu 3, B-1348 Louvain-la-Neuve (Belgium). boucekkine@core.ucl.ac.be

${ }^{\ddagger}$ Department of Economics, Universidad Complutense de Madrid. blmartin@ccee.ucm.es

$\S$ Department of Economics, Universitat de València and Université Catholique de Louvain. ramon.ruiz@uv.es 


\section{Introduction}

The Lucas-Uzawa model has been at the heart of macroeconomic research for many decades, and it is still widely considered as a fundamental benchmark for ongoing research programs in many fields, notably in economic development and growth theory (see for example d'Albis and Le Van, 2006). Two characteristics of the model makes it, however, nontrivial mathematically speaking: it is a two-sector model (with two controls and two state variables), which induces a much larger set of optimality conditions compared to the typical Ramsey model, and it is an endogenous growth model. Such models have the property of indeterminacy in the long-run variables' levels. A substantial part of the literature dealing with Lucas-Uzawa is precisely devoted to get through these problems. Important contributions in this topic are Caballé and Santos (1993), Xie (1994) and Benhabib and Perli (1994). None of these papers solves the model completely but they certainly allow to get an accurate picture of the mechanisms at work in the model. With the exception of Xie who solves explicitly a very special parametric case of the model, the other authors build on a transformed model with a lower dimension (3 against 4 in the original model) to overcome the problem of indeterminacy in levels. Typically, the (three) variables considered in the auxiliary models are the ratios physical to human capital and consumption to physical capital, and the fraction of human capital devoted to the final good sector. The same approach is taken in textbooks (see Barro and Sala-i-Martin, 1995, chapter 5).

Boucekkine and Ruiz-Tamarit (2007) have proposed a new method inspired from mathematical physics. Precisely, they show that the Lucas-Uzawa model can be explicitly solved for all variables in level using Gaussian hypergeometric functions. These solutions paths in levels have been shown to exhibit some specific non-monotonicities, a feature let in the dark in the traditional literature. However, the essential contribution of the paper is to supply the solution paths in closed form for any further exploration of the global dynamics of all the variables, a task which was considered as unrealistic for a long time. In this note, we show how the new approach can be advantageously used to dig deeper in the transition dynamics of the model, and notably in its imbalance effects.

Imbalance effects refer to the transition dynamics of a model when its state variables start below or above the corresponding long-run equilibrium value (see Barro and Sala-iMartin, 1995, chapter 5, and Mulligan and Sala-i-Martin, 1993).The economy is initially out of equilibrium, and if the adequate stability conditions are met, it has to come back after a certain adjustment phase. Admittedly, such a topic is better appraised and more interesting in a global framework, that is, by allowing the economy to start arbitrarily far from equilibrium. Unfortunately, the traditional method is based on linearization around steady-states, which disqualify it for any global inspection.

In the Lucas-Uzawa model, the imbalance effect story has been quickly reduced to the transition dynamics of the model when the ratio physical to human capital stocks is initially below or above the corresponding long-run value. This might make sense. On the theoretical ground, however, very few papers have been devoted to clarify this point. Caballé and Santos (1993) and Ortigueira (1998) are among the very few exceptions. In such a literature, it is typically shown that in the so-called normal parametric case and 
only in this case, ${ }^{1}$ the human capital stock should always increase at a rate higher than the long-run counterpart if the ratio physical to human capital stocks starts above the corresponding long-run value. That is, the initially scarce factor should be accumulated at a higher pace with respect to its long run balanced path. There are two important aspects in this analysis that could be easily improved within the new methodological setup designed by Boucekkine and Ruiz-Tamarit. First of all, and at almost zero cost, the properties can be established for all variables in level, and for arbitrary initial conditions, which is a key advantage given the specific issue investigated. Second, one can have a much more complete story of imbalance effects. Traditionally the analysis is restricted to the dynamics of human capital accumulation. What about physical capital? Suppose the economy starts with a ratio physical to human capital stocks above the corresponding long-run value. Then even though this ratio decreases monotonically to its long-run equilibrium value, physical capital can in principle either decrease or increase (at a rate lower than human capital) at least for some bounded time interval. Moreover, in the case the stock of physical grows from the beginning, there is no guarantee that it will grow at a rate lower than its long-run counterpart. Therefore, it is not clear at all a priori whether the stock of physical capital will have the same kind of behaviour as human capital, specially because, as established by Boucekkine and Ruiz-Tamarit (2007), such variable may exhibit non-monotonic paths in contrast to human capital stock. We will extend the theoretical analysis of imbalance effects to physical capital, and we will highlight an important asymmetry between physical and human capital. Precisely, we will show that whatever the parametric case considered, physical capital stock always grows at a rate lower than its long-run counterpart when the ratio physical to human capital is above its long run value.

The next section reminds the main equations involved in the Lucas-Uzawa model and gives the closed-form solutions detected by Boucekkine and Ruiz-Tamarit. Section 3 revisits the imbalance effects properties with the new approach.

\section{The optimal control problem and the Boucekkine- Ruiz-Tamarit closed-form solution}

\section{$2.1 \quad$ The model}

The problem is standard in economic growth (Lucas, 1988, for example). The economy is populated with many identical, rational agents, choosing the controls $c(t)$, consumption per capita, and $u(t) \forall t \geq t_{0}$, the fraction of non-leisure time devoted to goods production, which solve the dynamic optimization problem

$$
\max \int_{0}^{\infty} \frac{c(t)^{1-\sigma}-1}{1-\sigma} N(t) e^{-\rho t} d t
$$

subject to

$$
\dot{K}(t)=A K(t)^{\beta}(u(t) N(t) h(t))^{1-\beta}-\pi K(t)-N(t) c(t)
$$

\footnotetext{
${ }^{1}$ We shall be very clear about the meaning of a normal parameterization in Section 3 .
} 


$$
\begin{gathered}
\dot{h}(t)=\delta(1-u(t)) h(t)-\theta h(t), \\
K(0)=K_{0}, \quad h(0)=h_{0}, \quad N(0)=N_{0}, \\
c(t) \geqslant 0, \quad u(t) \in[0,1], \quad K(t) \geqslant 0, \quad h(t) \geqslant 0 .
\end{gathered}
$$

The considered instantaneous utility function is standard, with $\sigma^{-1}>0$ representing the constant elasticity of intertemporal substitution. Population at time $t$ is $N(t)$, which is assumed to grow at a constant exogenously given rate $n$. Parameter $\rho$ is the rate of time preference or discount rate. We assume $\rho>n . h(t)$ is the human capital level. The output, $Y(t)$, which may be allocated to consumption or to physical capital accumulation depends on the capital stock, $K(t)$, and the effective work force, $u(t) N(t) h(t)$. Parameter $\beta$ is the elasticity of output with respect to physical capital. The efficiency parameter $A$ represents the constant technological level in the goods sector of this economy. The efficiency parameter $\delta$ represents the constant technological level in the educational sector. Both physical and human capital depreciate at constant rates, which are $\pi \geqslant 0$ and $\theta \geqslant 0$, respectively. We shall also assume that $\delta+n>\theta+\rho$ for positive (long run) growth to arise, as it will be transparent later.

The current value Hamiltonian associated with the previous intertemporal optimization problem is

$$
\begin{gathered}
H^{c}\left(K, h, \vartheta_{1}, \vartheta_{2}, c, u ; A, \sigma, \beta, \delta, \pi, \theta,\{N(t): t \geq 0\}\right)= \\
=\frac{c^{1-\sigma}-1}{1-\sigma} N+\vartheta_{1}\left[A K^{\beta}(u N h)^{1-\beta}-\pi K-N c\right]+\vartheta_{2}[\delta(1-u) h-\theta h],
\end{gathered}
$$

where $\vartheta_{1}$ and $\vartheta_{2}$ are the co-state variables for $K$ and $h$, respectively.

The first order necessary conditions are

$$
\begin{gathered}
c^{-\sigma}=\vartheta_{1}, \\
\vartheta_{1}(1-\beta) A K^{\beta}(u N h)^{-\beta} N=\vartheta_{2} \delta,
\end{gathered}
$$

the Euler equations

$$
\begin{gathered}
\dot{\vartheta}_{1}=(\rho+\pi) \vartheta_{1}-\vartheta_{1} \beta A K^{\beta-1}(u N h)^{1-\beta}, \\
\dot{\vartheta}_{2}=(\rho+\theta) \vartheta_{2}-\vartheta_{1}(1-\beta) A K^{\beta}(u N)^{1-\beta} h^{-\beta}-\vartheta_{2} \delta(1-u),
\end{gathered}
$$

the dynamic constraints

$$
\begin{gathered}
\dot{K}=A K^{\beta}(u N h)^{1-\beta}-\pi K-N c, \\
\dot{h}=\delta(1-u) h-\theta h,
\end{gathered}
$$

the boundary conditions $K_{0}, h_{0}$, and the transversality conditions

$$
\begin{aligned}
& \lim _{t \rightarrow \infty} \vartheta_{1} K \exp \{-\rho t\}=0, \\
& \lim _{t \rightarrow \infty} \vartheta_{2} h \exp \{-\rho t\}=0 .
\end{aligned}
$$


From (3) and (4) we can express the control functions, $c$ and $u$ in terms of the other variables. These conditions also imply that, at any finite date, $\vartheta_{1} \neq 0$ and $\vartheta_{2} \neq 0$. After substituting these expressions into equations (5)-(8), we obtain the following four dimensional system, in terms of state and co-state variables only,

$$
\begin{gathered}
\dot{\vartheta}_{2}=-(\delta-\rho-\theta) \vartheta_{2}, \\
\dot{\vartheta}_{1}=(\rho+\pi) \vartheta_{1}-\psi_{1}(t) \vartheta_{1}^{\frac{1}{\beta}}, \\
\dot{K}=\psi_{2}(t) K-\psi_{3}(t), \\
\dot{h}=(\delta-\theta) h-\psi_{4}(t),
\end{gathered}
$$

where

$$
\begin{gathered}
\psi_{1}(t)=\beta A\left(\frac{(1-\beta) A}{\delta}\right)^{\frac{1-\beta}{\beta}} N^{\frac{1-\beta}{\beta}} \vartheta_{2}^{-\frac{1-\beta}{\beta}}, \\
\psi_{2}(t)=A\left(\frac{(1-\beta) A}{\delta}\right)^{\frac{1-\beta}{\beta}} N^{\frac{1-\beta}{\beta}}\left(\frac{\vartheta_{1}}{\vartheta_{2}}\right)^{\frac{1-\beta}{\beta}}-\pi, \\
\psi_{3}(t)=N \vartheta_{1}^{-\frac{1}{\sigma}}, \\
\psi_{4}(t)=\delta\left(\frac{(1-\beta) A}{\delta}\right)^{\frac{1}{\beta}} N^{\frac{1-\beta}{\beta}}\left(\frac{\vartheta_{1}}{\vartheta_{2}}\right)^{\frac{1}{\beta}} K .
\end{gathered}
$$

These equations, together with the initial conditions, $K_{0}$ and $h_{0}$, and the transversality conditions (9) and (10) constitute the dynamic system which drives the economy over time. This dynamic system can be recursively solved in closed form. Boucekkine and Ruiz-Tamarit (2007) show that such a system can be solved explicitly without resorting to any dimension reduction.

\subsection{Closed-form solutions}

Boucekkine and Ruiz-Tamarit (2007) use Gaussian hypergeometric functions ${ }_{2} F_{1}(a, b, c ; z)$ in their closed-form solution. Some basic definitions and properties of such functions are reported in the appendix. Precisely, they show that the solutions of the system (11)(14) can be written in terms of Gaussian hypergeometric functions in their Euler integral representation.

More precisely, let us define the hypergeometric function

$$
\begin{gathered}
{ }_{2} F_{1}(t)={ }_{2} F_{1}\left(a, b, c ;\left(1-\frac{\delta+n+\pi-\theta}{\epsilon}\left(\frac{\vartheta_{1}(0)}{\vartheta_{2}(0)}\right)^{-\frac{1-\beta}{\beta}}\right) \exp \left\{-\frac{(1-\beta)(\delta+n+\pi-\theta)}{\beta} t\right\}\right), \\
{ }_{2} F_{1}(0)={ }_{2} F_{1}\left(a, b, c ; 1-\frac{\delta+n+\pi-\theta}{\epsilon}\left(\frac{\vartheta_{1}(0)}{\vartheta_{2}(0)}\right)^{-\frac{1-\beta}{\beta}}\right),
\end{gathered}
$$


with

$$
\begin{gathered}
\epsilon=\beta A\left(\frac{(1-\beta) A N_{0}}{\delta}\right)^{\frac{1-\beta}{\beta}}, \\
a=-\frac{(\delta+n+\pi-\theta)(\beta-\sigma)-\beta(\rho+\pi-n \sigma-\pi \sigma)}{\sigma(\delta+n+\pi-\theta)(1-\beta)}, \quad b=-\frac{\beta-\sigma}{\sigma(1-\beta)}, \quad c=1+a .
\end{gathered}
$$

Then the optimal trajectory of physical capital is given by (See Boucekkine and RuizTamarit, 2007, Proposition 4):

$$
\begin{gathered}
K=K_{0}\left(\frac{\vartheta_{1}(0)}{\vartheta_{2}(0)}\right)^{\frac{1}{\beta}}\left(\frac{\epsilon}{\delta+n+\pi-\theta}\right)^{\frac{1}{1-\beta}} \frac{{ }_{2} F_{1}(t)}{{ }_{2} F_{1}(0)} \\
\cdot \exp \left\{\frac{(\delta+n+\pi-\theta)(\beta-\sigma)-\beta(\rho+\pi-n \sigma)}{\beta \sigma} t\right\} \\
\cdot\left[-1+\exp \left\{\frac{(1-\beta)(\delta+n+\pi-\theta)}{\beta} t\right\}+\frac{\delta+n+\pi-\theta}{\epsilon}\left(\frac{\vartheta_{1}(0)}{\vartheta_{2}(0)}\right)^{-\frac{1-\beta}{\beta}}\right]^{\frac{1}{1-\beta}} .
\end{gathered}
$$

Similarly, one can define the auxiliary hypergeometric function for the solution path of human capital

$$
\begin{gathered}
{ }_{2} \widetilde{F}_{1}(t)={ }_{2} F_{1}\left(\widetilde{a}, b, c ;\left(1-\frac{\delta+n+\pi-\theta}{\epsilon}\left(\frac{\vartheta_{1}(0)}{\vartheta_{2}(0)}\right)^{-\frac{1-\beta}{\beta}}\right) \exp \left\{-\frac{(1-\beta)(\delta+n+\pi-\theta)}{\beta} t\right\}\right), \\
{ }_{2} \widetilde{F}_{1}(0)={ }_{2} F_{1}\left(\widetilde{a}, b, c ; 1-\frac{\delta+n+\pi-\theta}{\epsilon}\left(\frac{\vartheta_{1}(0)}{\vartheta_{2}(0)}\right)^{-\frac{1-\beta}{\beta}}\right),
\end{gathered}
$$

with

$$
\widetilde{a}=a-1=-\frac{(\delta+n+\pi-\theta) \beta(1-\sigma)-\beta(\rho+\pi-n \sigma-\pi \sigma)}{\sigma(\delta+n+\pi-\theta)(1-\beta)} .
$$

Then the optimal human capital trajectory should fulfill (See Proposition 5 in Boucekkine and Ruiz-Tamarit, 2007):

$$
h=h_{0} \frac{{ }_{2} \widetilde{F}_{2}(t)}{{ }_{2} \widetilde{F}_{1}(0)} \exp \left\{\frac{\delta+n-\theta-\rho}{\sigma} t\right\} ;
$$

It should be noted that (19) and (20) characterize completely the solution paths for both stocks of capital provided that the shadow price values $\vartheta_{1}(0)$ and $\vartheta_{2}(0)$ be identified. Boucekkine and Ruiz-Tamarit (2007) also show that in addition to some traditional parametric restrictions yielding, among others, $a>1$, the transversality conditions impose the following constraints on the initial shadow price values (Proposition 3 in Boucekkine and Ruiz-Tamarit, 2007)

$$
\begin{gathered}
\frac{K_{0}}{{ }_{2} F_{1}(0)}\left(\frac{\vartheta_{1}(0)}{\vartheta_{2}(0)}\right)^{\frac{1}{\beta}}=-\frac{\sigma \beta N_{0} \vartheta_{2}(0)^{-\frac{1}{\sigma}}\left(\frac{\delta+n+\pi-\theta}{\epsilon}\right)^{\frac{\sigma-\beta}{\sigma(1-\beta)}}}{(\delta+n+\pi-\theta)(\beta-\sigma)-\beta(\rho+\pi-n \sigma-\pi \sigma)}, \\
\frac{{ }_{2} F_{1}(0)}{{ }_{2} \widetilde{F}_{1}(0)}=\frac{(1-\beta) \epsilon \sigma}{-((\delta-\theta)(1-\sigma)+n-\rho) \beta} \frac{K_{0}}{h_{0}}\left(\frac{\vartheta_{1}(0)}{\vartheta_{2}(0)}\right)^{\frac{1}{\beta}} .
\end{gathered}
$$




\section{Global dynamics and imbalance effects}

We start with a brief discussion of the concept of imbalance effects. For the sake of simplicity, we assume constant population $(n=0)$, and $N_{0}=1$.

\subsection{Imbalance effects: revisiting the concept}

The imbalance effects have been so far deeply studied in two-sector endogenous growth models, especially thanks to the contributions of Sala-i-Martin and his co-authors. Nonetheless, the analysis has been so far mostly computational, with some few exceptions like Caballé and Santos (1993) and Ortigueira (1998). Using the 3-dimensional transformed Lucas-Uzawa model, and linearizing around the corresponding steady state, these authors have noticed that the transition dynamics depend strongly on the value of the capital share in the final good production function, $\beta$, and on the inverse of the intertemporal elasticity of substitution, $\sigma$. Ortigueira, in page 330, defines the normal case as follows:

Definition 1 If the economy starts with a higher physical-human capital ratio than that of the stationary solution, then the (short-run) rate of human capital accumulation is higher than that of the long-run equilibrium. That is,

$$
\frac{K_{0}}{h_{0}}>\left(\frac{\bar{K}}{h}\right) \Rightarrow \frac{\dot{h}}{h}>\frac{\dot{\bar{h}}}{\bar{h}} .
$$

Then, he proves that the economy belongs to the normal case if and only if $\beta<\sigma$ (Corollary 1, page 332). The same result was obtained by Caballé and Santos (1993). The proof of such a property within the traditional approach is definitely easy since the growth rate of human capital is exclusively driven by $1-u$ (i.e., the fraction of human capital channeled into the education sector), and the variable $u$ is one of the three variables considered in the 3-dimensional auxiliary model. The transition dynamics of physical capital are then supplied numerically.

As argued in the introduction, we do believe that a more global appraisal of imbalance effects should take into account the two state variables, physical and human capital. The fact that the short-run rate of human capital accumulation is higher than that of the long-run equilibrium does not guarantee that the short-run rate of physical capital accumulation is lower than that of the long-run equilibrium. We shall therefore consider a more general definition of the normal case as follows:

Definition 2 If the economy starts with a higher physical-human capital ratio than that of the stationary solution, then the short-run rate of human capital accumulation is higher than that of the long-run equilibrium, and the short-run rate of physical capital accumulation is lower than that of the long-run equilibrium. That is,

$$
\frac{K_{0}}{h_{0}}>\left(\frac{\bar{K}}{h}\right) \Rightarrow \frac{\dot{h}}{h}>\frac{\dot{\bar{h}}}{\bar{h}},
$$


and

$$
\frac{K_{0}}{h_{0}}>\left(\frac{\bar{K}}{h}\right) \Rightarrow \frac{\dot{K}}{K}<\frac{\dot{\bar{K}}}{\bar{K}}
$$

We are simply stating that in case of initial imbalance, the relatively scarce variable should grow at a higher rate than in the long-run, while the variable relatively in excess should have the opposite behaviour. The next section is first devoted to show that the parametric characterization of Caballé and Santos (1993) and Ortigueira (1998) can be extended to any initial conditions (global dynamics). Then, we will analyze the imbalance effects in the light of the stricter concept defined just above and identify the announced asymmetry.

\subsection{Human capital dynamics}

First, using equations (19) and (20) respectively, one can identify the long-run balanced growth paths of physical and human capital stocks. Indeed:

$$
\bar{K}=\frac{K_{0}}{{ }_{2} F_{1}(0)}\left(\frac{\vartheta_{1}(0)}{\vartheta_{2}(0)}\right)^{\frac{1}{\beta}}\left(\frac{\epsilon}{\delta+n+\pi-\theta}\right)^{\frac{1}{1-\beta}} \exp \left\{\frac{\delta+n-\theta-\rho+n \sigma}{\sigma} t\right\},
$$

and

$$
\bar{h}=\frac{h_{0}}{{ }_{2} \widetilde{F}_{1}(0)} \exp \left\{\frac{\delta+n-\theta-\rho}{\sigma} t\right\} .
$$

Under the constant population assumption, we set $\Theta=\frac{(1-\beta) \epsilon \sigma}{-((\delta-\theta)(1-\sigma)-\rho) \beta}, \eta=\frac{\vartheta_{1}(0)}{\vartheta_{2}(0)}, \epsilon=$ $\beta A\left(\frac{(1-\beta) A}{\delta}\right)^{\frac{1-\beta}{\beta}}$, and $\Lambda=\frac{\delta+\pi-\theta}{\epsilon}$. Then, one can rewrite the balanced growth paths as

$$
\begin{gathered}
\bar{K}=\frac{K_{0}}{{ }_{2} F_{1}(0)} \eta^{\frac{1}{\beta}} \frac{1}{\Lambda^{\frac{1}{1-\beta}}} \exp \left\{\frac{\delta-\theta-\rho}{\sigma} t\right\}, \\
\bar{h}=\frac{h_{0}}{{ }_{2} \widetilde{F}_{1}(0)} \exp \left\{\frac{\delta-\theta-\rho}{\sigma} t\right\} .
\end{gathered}
$$

This allows to write the long-run ratio physical to human capital as

$$
\left(\frac{\bar{K}}{h}\right)=\frac{K_{0}}{h_{0}} \frac{{ }_{2} \widetilde{F}_{1}(0)}{{ }_{2} F_{1}(0)} \frac{1}{\Lambda^{\frac{1}{1-\beta}}} \eta^{\frac{1}{\beta}} .
$$

Therefore, if the economy starts from above, that is $\frac{K_{0}}{h_{0}}>\left(\frac{\bar{K}}{h}\right)$, we have

$$
1>\frac{{ }_{2} \widetilde{F}_{1}(0)}{{ }_{2} F_{1}(0)} \frac{1}{\Lambda^{\frac{1}{1-\beta}}} \eta^{\frac{1}{\beta}}
$$

or, using the transversality condition (22),

$$
\frac{K_{0}}{h_{0}}>\frac{1}{\Theta} \frac{1}{\Lambda^{\frac{1}{1-\beta}}} .
$$


We shall now study the growth rate of human capital using the closed-form solution of this variable. Comparing equation (20) with (24), one gets immediately $h$ as a function of $\bar{h}$ and ${ }_{2} \widetilde{F}_{1}(t)$, which is very useful for the inspection of imbalance effects,

$$
h={ }_{2} \widetilde{F}_{1}(t) \bar{h} .
$$

Indeed, it follows immediately that

$$
\frac{\dot{h}}{h}=\frac{{ }_{2} \dot{\widetilde{F}}_{1}(t)}{{ }_{2} \widetilde{F}_{1}(t)}+\frac{\dot{\bar{h}}}{\bar{h}}
$$

and finally we may conclude

$$
\frac{\dot{h}}{h}>\frac{\dot{h}}{\bar{h}} \Longleftrightarrow \frac{{ }_{2} \dot{\widetilde{F}}_{1}(t)}{{ }_{2} \widetilde{F}_{1}(t)}>0 .
$$

It is then almost trivial to obtain a global counterpart of the traditional local result on the parametric characterization of the normal case:

Proposition 1 If the economy is characterized by $\sigma>\beta$, and only in this case, we have

$$
\frac{K_{0}}{h_{0}}>\left(\frac{\bar{K}}{h}\right) \Rightarrow \frac{\dot{h}}{h}>\frac{\dot{\bar{h}}}{\bar{h}} \text {. }
$$

Proof: The proof is somewhat immediate after the computation of the growth rate of the hypergeometric function ${ }_{2} \widetilde{F}_{1}(t)$. The algebra needed for such a computation is trivial for special functions users and it is reported in the Appendix. We get

$$
\frac{{ }_{2} \dot{\widetilde{F}}_{1}(t)}{{ }_{2} \widetilde{F}_{1}(t)}>0 \Longleftrightarrow \frac{b(a-1)}{a+1} \dot{z}(t) \frac{{ }_{2} F_{1}(b+1, a, a+2 ; z(t))}{{ }_{2} F_{1}(b, a-1, a+1 ; z(t))}>0,
$$

with $z(t)=z_{0} \exp \left\{-\frac{(1-\beta)(\delta+\pi-\theta)}{\beta} t\right\}$, and $z_{0}=\left(1-\Lambda \eta^{-\frac{1-\beta}{\beta}}\right)$. Boucekkine and RuizTamarit (2007) prove that $z_{0}<0$ if $\frac{K_{0}}{h_{0}}>\left(\frac{\bar{K}}{h}\right)$, and $0<z_{0}<1$ if $\frac{K_{0}}{h_{0}}<\left(\frac{\bar{K}}{h}\right)$, while if $\frac{K_{0}}{h_{0}}=\left(\frac{\bar{K}}{h}\right)$, we have $z_{0}=0$ (See Lemma 2, Lemma 3 and Proposition 7 in Boucekkine and Ruiz-Tamarit, 2007). Therefore, in the case studied in this proposition, $z_{0}<0$. Because

$$
\dot{z}(t)=-\frac{(1-\beta)(\delta+\pi-\theta)}{\beta} z_{0} \exp \left\{-\frac{(1-\beta)(\delta+\pi-\theta)}{\beta} t\right\},
$$

and $\delta+\pi>\theta$, since $\delta>\theta+\rho$ for positive growth, ${ }^{2}$ it follows that $\dot{z}(t)$ is strictly positive. Now, it is sufficient to look at the expression of $\frac{2 \dot{\widetilde{F}}_{1}(t)}{{ }_{2} \widetilde{F}_{1}(t)}$ just above to get the result. Since $a>1$, and the involved hypergeometric functions ${ }_{2} F_{1}(b+1, a, a+2 ; z(t))$

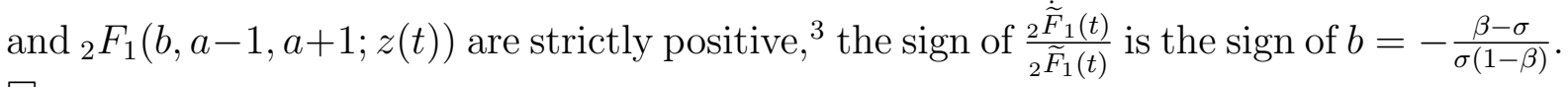

\footnotetext{
${ }^{2}$ See for example equation (20).

${ }^{3}$ This is because they admit the Euler integral representation.
} 


\subsection{The dynamics of physical capital}

We now move to the dynamics of physical capital, and provide a similar global analysis as for human capital in accordance with our interpretation of imbalance effects. Not surprisingly, the task proves more difficult than for human capital. However the algebra needed is by no way intractable. Recall the closed-form solution for physical capital given in (19),

$$
\begin{aligned}
K=K_{0} \eta^{1 / \beta} & \left(\frac{1}{\Lambda}\right)^{\frac{1}{1-\beta}} \frac{{ }_{2} F_{1}(t)}{{ }_{2} F_{1}(0)} \exp \left\{\frac{(\delta+\pi-\theta)(\beta-\sigma)-\beta(\rho+\pi)}{\beta \sigma} t\right\} \\
\cdot & {\left[-z_{0}+\exp \left\{\frac{(1-\beta)(\delta+\pi-\theta)}{\beta} t\right\}\right]^{\frac{1}{1-\beta}} . }
\end{aligned}
$$

Comparing with its balanced growth path, we can rewrite $K$ into a more manageable form:

$$
K(t)=\bar{K}(t){ }_{2} F_{1}(t) G(t),
$$

where

$$
G(t)=\exp \left\{\frac{-(\delta+\pi-\theta)}{\beta} t\right\}\left[-z_{0}+\exp \left\{\frac{(1-\beta)(\delta+\pi-\theta)}{\beta} t\right\}\right]^{\frac{1}{1-\beta}} .
$$

Then, the growth rate of physical capital is given by

$$
\frac{\dot{K}}{K}=\frac{\dot{\bar{K}}}{\bar{K}}+\frac{{ }_{2} \dot{F}_{1}(t)}{{ }_{2} F_{1}(t)}+\frac{\dot{G}(t)}{G(t)}
$$

Accordingly, forphysical capital to behave inaccordance with the normal case defined in Section 3.1, we need to assess the inequality

$$
\frac{{ }_{2} \dot{F}_{1}(t)}{{ }_{2} F_{1}(t)}+\frac{\dot{G}(t)}{G(t)}<0
$$

when the economy starts with a physical to human capital ratio above its long-run counterpart. We shall first prove that if we are in the normal parametric case identified by Caballé and Santos, then such an inequality is indeed fulfilled.

Proposition 2 When the economy starts with a higher physical-human capital ratio than that of the stationary solution and the condition $\beta<\sigma$ is checked, the short-run rate of physical capital accumulation is lower than that of the long-run equilibrium.

Proof: Using the Euler integral representation of the hypergeometric function ${ }_{2} F_{1}(t)$ one finds, after some trivial algebra,

$$
\frac{{ }_{2} \dot{F}_{1}(t)}{{ }_{2} F_{1}(t)}=-b z_{0} \frac{(1-\beta)(\delta+\pi-\theta)}{\beta} \exp \left\{-\frac{(1-\beta)(\delta+\pi-\theta)}{\beta} t\right\} \frac{a}{a+1} \frac{{ }_{2} F_{1}(b+1, a+1, a+2 ; z(t))}{{ }_{2} F_{1}(b, a, a+1 ; z(t))}
$$


where $z(t)=z_{0} \exp \left\{-\frac{(1-\beta)(\delta+\pi-\theta)}{\beta} t\right\}$ as before. Note that $\frac{{ }_{2} \dot{F}_{1}(t)}{{ }_{2}(t)}$ is a positive function that tends to $-b z_{0} \frac{(1-\beta)(\delta+\pi-\theta)}{\beta} \frac{a}{a+1} \frac{2 F_{1}\left(b+1, a+1, a+2 ; z_{0}\right)}{{ }_{2} F_{1}\left(b, a, a+1 ; z_{0}\right)}$ when $t \rightarrow 0$, and tends to 0 when $t \rightarrow \infty$. On the other hand,

$$
\frac{\dot{G}(t)}{G(t)}=\frac{(\delta+\pi-\theta)}{\beta} \frac{z_{0}}{-z_{0}+\exp \left\{\frac{(1-\beta)(\delta+\pi-\theta)}{\beta} t\right\}},
$$

which is a negative function that tends to $\frac{\delta+\pi-\theta}{\beta} \frac{z_{0}}{1-z_{0}}$ when $t \rightarrow 0$, and tends to 0 when $t \rightarrow \infty$. So unfortunately, the sign of $\frac{{ }_{2} \dot{F}_{1}(t)}{{ }_{2} F_{1}(t)}+\frac{\dot{G}(t)}{G(t)}$ is undetermined at this stage, and we have to dig deeper. Using the two previous expressions we find that ${ }^{4}$

$$
\frac{{ }_{2} \dot{F}_{1}(t)}{{ }_{2} F_{1}(t)}+\frac{\dot{G}(t)}{G(t)}=-\frac{(\delta+\pi-\theta) z}{\beta}\left[\frac{b a(1-\beta)}{a+1} \frac{{ }_{2} F_{1}(b+1, a+1, a+2 ; z)}{{ }_{2} F_{1}(b, a, a+1 ; z)}-\frac{1}{1-z}\right] .
$$

Applying formula (15.2.1) from Abramowitz and Stegun (1972), we can rewrite the previous expression in a more advantageous way

$$
\frac{{ }_{2} \dot{F}_{1}(t)}{{ }_{2} F_{1}(t)}+\frac{\dot{G}(t)}{G(t)}=-\frac{(\delta+\pi-\theta)(1-\beta) z}{\beta}\left[\frac{\frac{d\left[{ }_{2} F_{1}(b, a, a+1 ; z)\right]}{d z}}{{ }_{2} F_{1}(b, a, a+1 ; z)}-\frac{1}{1-\beta} \frac{1}{1-z}\right] .
$$

Given that $\delta+\pi>\theta$, and $z<0$ because $z_{0}<0$ when the initial physical to human capital ratio is above its long-run value (see Proof of Proposition 1), it follows that the short-run rate of physical capital accumulation is lower than that of the long-run equilibrium if and only if

$$
\frac{\frac{d\left[{ }_{2} F_{1}(b, a, a+1 ; z)\right]}{d z}}{{ }_{2} F_{1}(b, a, a+1 ; z)}-\frac{1}{1-\beta} \frac{1}{1-z}<0, \quad \forall z<0 .
$$

Integrating the previous inequality between $z(<0)$ and 0 , we obtain that the latter inequality is indeed equivalent to

$$
{ }_{2} F_{1}(b, a, a+1 ; z)-(1-z)^{-\frac{1}{1-\beta}}>0, \quad \forall z<0 .
$$

We denote by $H(z)$ the function defined as the expression on the left hand side of the previous inequality. Then, the short-run rate of physical capital accumulation is lower than that of the long-run equilibrium if and only if $H(z)$ is positive $\forall z<0$.

Suppose $\sigma>\beta$ or $b>0$. We shall prove that in such a parametric case: $(i) H(0)=0$, $H^{\prime}(0)<0$, and the limit of $H(z)$ when $z$ tends to $-\infty$ is zero, and $(i i) H^{\prime}(z)$ has a unique root. These conditions are sufficient for $H(z)$ positive $\forall z<0$.

Note that $H(0)={ }_{2} F_{1}(b, a, a+1 ; 0)-1=0$. By continuity, $H(z) \rightarrow 0$ when $z \rightarrow 0$. This result implies, in our dynamical model, that $\frac{\dot{K}}{K}$ tends to $\frac{\dot{\bar{K}}}{\bar{K}}$ when $t$ tends to $\infty$. Moreover,

$$
H^{\prime}(z)=\frac{a b}{1+a}{ }_{2} F_{1}(b+1, a+1, a+2 ; z)-\frac{1}{1-\beta}(1-z)^{-\frac{2-\beta}{1-\beta}},
$$

\footnotetext{
${ }^{4}$ For the sake of simplicity, from now on we have removed the temporal index corresponding to the variable $z$.
} 
which implies that

$$
H^{\prime}(0)=\frac{a b}{1+a}-\frac{1}{1-\beta}=\frac{1}{1-\beta}\left(\frac{a}{a+1} \frac{\sigma-\beta}{\sigma}-1\right)<0 .
$$

In order to prove that $H(z)$ goes to zero when $z$ goes to $-\infty$, we use the Euler integral representation of the hypergeometric function ${ }_{2} F_{1}(b, a, a+1 ; z)$ to find an upper bound going to zero when $z$ goes to $-\infty$. This will be enough to conclude since ${ }_{2} F_{1}($.$) is positive$ $\forall z<0$ and the limit of $(1-z)^{-\frac{1}{1-\beta}}$ is zero when $z$ tends to $-\infty$. Indeed,

$$
{ }_{2} F_{1}(b, a, a+1 ; z)=\frac{\Gamma(a+1)}{\Gamma(a) \Gamma(1)} \int_{0}^{1} v^{a-1}(1-v z)^{-b} d v \leq a \int_{0}^{1}(1-v z)^{-b} d v=\frac{a}{(1-b) z}-\frac{a(1-z)^{1-b}}{(1-b) z} .
$$

The limit of the first term on the right hand side when $z$ tends to $-\infty$ is zero, but it is also zero the limit of the second term when $b>0$. The latter being obvious if $b>1$, and immediate if $1>b>0$ after application of the l' Hôpital's rule. This allows already to conclude that $H(z)$ goes to zero when $z$ goes to $-\infty$ under $b>0$.

Now, recall (35) denoting $h_{1}(z)=\frac{a b}{1+a}{ }_{2} F_{1}(b+1, a+1, a+2 ; z)$ and $h_{2}(z)=\frac{1}{1-\beta}(1-$ $z)^{-\frac{2-\beta}{1-\beta}}$ so that $H^{\prime}(z)=h_{1}(z)-h_{2}(z)$. It can be easily shown that both functions $h_{1}($. and $h_{2}($.$) are both strictly increasing and strictly convex for z<0$. Moreover, one can readily see that both functions cannot intersect in more than 2 points if any intersection. We shall show that the two functions do always intersect in a single point. Because of $H^{\prime}(0)<0$, we know that $h_{1}(0)<h_{2}(0)$. We now show that this inequality should be reverted for $z$ big enough (in absolute value). Indeed, one can straightforwardly find a lower bound for $h_{1}(z)$ because

$$
\begin{gathered}
{ }_{2} F_{1}(b+1, a+1, a+2 ; z)= \\
(1+a) \int_{0}^{1} v^{a}(1-v z)^{-b-1} d v \geq(1+a)(1-z)^{-b-1} \int_{0}^{1} v^{a} d v=(1-z)^{-b-1} .
\end{gathered}
$$

Using this result we can see that

$$
\frac{h_{1}(z)}{h_{2}(z)} \geq \frac{a b}{1+a}(1-\beta)(1-z)^{-b-1+\frac{2-\beta}{1-\beta}} .
$$

Then, omitting the constant terms, it follows that the ratio $h_{1}(z)$ to $h_{2}(z)$ behaves like the function $(1-z)^{\phi}$, with $\phi=-b-1+\frac{2-\beta}{1-\beta}=\frac{1}{1-\beta}-b>0$ as shown above. Hence, $h_{1}(z)$ is necessarily above function $h_{2}(z)$ for $z$ large enough (in absolute value). This implies not only that $H^{\prime}(z)=0$ has a root but also that it can not have two.

It should be noted that the statement of Proposition 2 is a sufficient condition for normal dynamics in $K: b>0$ is such a condition. Instead, for human capital Proposition 1 shows that normal dynamics are obtained if and only if $b>0$. Actually, one can go a step further and show that whatever the value of $b$, we get always normal dynamics for $K$. This features a kind of asymmetry between the two capital stocks, which is hidden in the theoretical literature taking the traditional approach. ${ }^{5}$ We prove this property next.

\footnotetext{
${ }^{5}$ It is fair to recognize that this feature is neatly pointed out in the computational literature à la Sala-i-Martin.
} 
Proposition 3 When the economy starts with a higher physical-human capital ratio than that of the stationary solution, the short-run rate of physical capital accumulation is lower than that of the long-run equilibrium whatever the sign of $\sigma-\beta$.

Proof: The proof is trivial using the previous proposition and equation (35). If $b \leq 0$, then $H^{\prime}(z)<0$ for all $z \leq 0$. Since $H(0)=0$, this means that $H(z)$ is always positive for $z$ negative.

As announced, we have thus shown that in contrast to human capital, physical capital stock grows at a rate greater than its long-run counterpart when the ratio physical to human capital is below its long run value whatever the position of the capital share in the production of physical good with respect to the inverse of the elasticity of intertemporal substitution in consumption.

\section{References}

[1] M. Abramowitz and I. A. Stegun, Handbook of Mathematical Functions. Dover Publications (1972).

[2] R. Barro and X. Sala-i-Martin, Economic Growth. McGraw-Hill (1995)

[3] J. Benhabib and R. Perli, Uniqueness and Indeterminacy: On the Dynamics of Endogenous Growth, Journal of Economic Theory 63 (1994), 113-142.

[4] R. Boucekkine and J. R. Ruiz-Tamarit, Special Functions of the Study of Economic Dynamics: The Case of the Lucas-Uzawa Model, Journal of Mathematical Economics, forthcoming, (2007).

[5] J. Caballé and M. S. Santos, On Endogenous Growth with Physical and Human Capital, Journal of Political Economy 101 (1993), 1042-1067.

[6] H. d'Albis and C. Le Van, Existence of a Competitive Equilibrium in the Lucas (1988) Model without Physical Capital, Journal of Mathematical Economics 42 (2006), 4655 .

[7] R. E. Lucas, Jr., On the Mechanics of Economic Development, Journal of Monetary Economics 22 (1988), 3-42.

[8] C. B. Mulligan and X. Sala-i-Martín, Transitional Dynamics in Two Sector Models of Endogenous Growth, Quarterly Journal of Economics 108 (1993), 739-773.

[9] S. Ortigueira, Fiscal Policy in an Endogenous Growth Model with Human Capital Accumulation, Journal of Monetary Economics 42 (1998), 323-355.

[10] D. Xie, Divergence in Economic Performance: Transitional Dynamics with Multiple Equilibria, Journal of Economic Theory 63 (1994), 97-112. 


\section{Appendix}

\section{The Gaussian hypergeometric function: definitions and the Euler integral representation}

Recall that the Gauss hypergeometric function (see Abramowitz and Stegun, 1972, or Temme, 1996), ${ }_{2} F_{1}(a, b, c ; z)$, with complex arguments $a, b, c$ and $z$, is given by the series

$$
{ }_{2} F_{1}(a, b, c ; z)=\sum_{n=0}^{\infty} \frac{(a)_{n}(b)_{n}}{(c)_{n}} \frac{z^{n}}{n !}
$$

where $(x)_{n}$ is the so-called Pochhammer symbol, defined by

$$
(x)_{n}=\frac{\Gamma(x+n)}{\Gamma(x)},
$$

where $\Gamma($.$) is the special function Gamma. One of the main properties of the Gauss$ hypergeometric function is that its circle of convergence is the unit circle. Fortunately, there are some ways to define it outside the unit circle, the Euler integral representation being the most practical continuation formula

$$
{ }_{2} F_{1}(a, b, c ; z)=\frac{\Gamma(c)}{\Gamma(b) \Gamma(c-b)} \int_{0}^{1} t^{b-1}(1-t)^{c-b-1}(1-t z)^{-a} d t
$$

when $\operatorname{Re}(c)>\operatorname{Re}(b)>0$ (see Abramowitz and Stegun, 15.3.1, page 558).

\section{Computation of the growth rate of the hypergeometric function ${ }_{2} \widetilde{F}_{1}(t)$ in Proposition 1}

Using the symmetry property in the arguments $a$ and $b$, and taking into account that $\widetilde{a}=a-1$ and $c=a+1$, we can rewrite ${ }_{2} \widetilde{F}_{1}(t)$ as

$$
{ }_{2} \widetilde{F}_{1}(t)={ }_{2} F_{1}(\widetilde{a}, b, c ; z(t))={ }_{2} F_{1}(a-1, b, a+1 ; z(t))={ }_{2} F_{1}(b, a-1, a+1 ; z(t)) .
$$

Since the previous hypergeometric function checks conditions under which the Euler integral representation holds $(a+1>a-1>0)$, we can rewrite it as

$$
\begin{gathered}
{ }_{2} F_{1}(\widetilde{a}, b, c ; z(t))={ }_{2} F_{1}(b, a-1, a+1 ; z(t))=\frac{\Gamma(a+1)}{\Gamma(a-1) \Gamma(2)} \int_{0}^{1} v^{a-2}(1-v)(1-v z(t))^{-b} d v= \\
a(a-1) \int_{0}^{1} v^{a-2}(1-v)(1-v z(t))^{-b} d v .
\end{gathered}
$$

The derivative of the previous expression with respect to $t$ is

$$
{ }_{2} \dot{\widetilde{F}}_{1}(t)=a(a-1) b \dot{z}(t) \int_{0}^{1} v^{a-1}(1-v)(1-v z(t))^{-b-1} d v .
$$


Since

$$
\begin{gathered}
\int_{0}^{1} v^{a-1}(1-v)(1-v z(t))^{-b-1} d v=\frac{\Gamma(a) \Gamma(2)}{\Gamma(a+2)}{ }_{2} F_{1}(b+1, a, a+2 ; z(t))= \\
=\frac{1}{a(a+1)}{ }_{2} F_{1}(b+1, a, a+2 ; z(t))
\end{gathered}
$$

then

$$
{ }_{2} \dot{\widetilde{F}}_{1}(t)=b \dot{z}(t) \frac{a-1}{a+1}{ }_{2} F_{1}(b+1, a, a+2 ; z(t)) .
$$

Finally, we get

$$
\frac{{ }_{2} \dot{\widetilde{F}}_{1}(t)}{{ }_{2} \widetilde{F}_{1}(t)}=b \dot{z}(t) \frac{a-1}{a+1} \frac{{ }_{2} F_{1}(b+1, a, a+2 ; z(t))}{{ }_{2} F_{1}(b, a-1, a+1 ; z(t))} .
$$

